ERR A T UM

Bernard Moussian · Achim Haecker - Thomas Laux

\title{
ZWILLE buffers meristem stability in Arabidopsis thaliana
}

Published online: 31 October 2003

(C) Springer-Verlag 2003

\section{Dev Genes Evol (2003) \\ DOI 10.1007/s00427-003-0359-z}

A version of this paper missing corrections made by the co-authors was by mistake submitted for print. While the data presented in the printed version are correct, several statements and citations were left uncorrected. The manuscript containing these corrections is available upon request from the corresponding author (bernard. moussian@tuebingen.mpg.de).

The online version of the original article can be found at http://dx.doi.org/10.1007/s00427-003-0359-z

B. Moussian $(-$ A. Haecker · T. Laux

Lehrstuhl für Entwicklungsgenetik,

Eberhard-Karl-Universität Tübingen,

Auf der Morgenstelle 1, 72074 Tübingen, Germany

e-mail: bernard.moussian@ @uebingen.mpg.de

Tel.: +49-7071-601492

Present address:

B. Moussian, Max-Planck-Institut für Entwicklungsbiologie,

Spemannstrasse 35,

72076 Tübingen, Germany

Present address:

A. Haecker, Department of Developmental Biology,

Arrhenius Laboratory E3, Stockholm University,

Svente Arrhenius Väg 16-18, 10691 Stockholm, Sweden

Present address:

T. Laux, Institut für Biologie III,

Universität Freiburg,

Schänzlestrasse 1, 79104 Freiburg, Germany 